\title{
Phase 1 study of MLN8054, a selective inhibitor of Aurora A kinase in patients with advanced solid tumors
}

\author{
E. Claire Dees ${ }^{1}$, Jeffrey R. Infante ${ }^{2}$, Roger B. Cohen ${ }^{3}$, Bert H. O'Neil ${ }^{1}$, Suzanne Jones ${ }^{2}$, \\ Margaret von Mehren ${ }^{3}$, Hadi Danaee ${ }^{4}$, Yih Lee ${ }^{4,{ }^{*}}$, Jeffrey Ecsedy $^{4}$, Mark Manfredi ${ }^{4}$, \\ Katherine Galvin ${ }^{4}$, Bradley Stringer ${ }^{4}$, Hua Liu ${ }^{4}$, Omar Eton ${ }^{4, \dagger}$, Howard Fingert ${ }^{4}$, and \\ Howard Burris ${ }^{2}$ \\ ${ }^{1}$ UNC Lineberger Comprehensive Cancer Center, Chapel Hill, NC \\ 2Sarah Cannon Research Institute, Nashville, TN \\ ${ }^{3}$ Fox Chase Cancer Center, Philadelphia, PA, USA \\ ${ }^{4}$ Millennium Pharmaceuticals, Inc., Cambridge, MA
}

\begin{abstract}
Purpose-Aurora A kinase is critical in assembly and function of the mitotic spindle. It is overexpressed in various tumor types and implicated in oncogenesis and tumor progression. This trial evaluated the dose-limiting toxicities (DLTs) and maximum tolerated dose (MTD) of MLN8054, a selective small-molecule inhibitor of Aurora A kinase.

Methods-In this first-in-human, dose-escalation study, MLN8054 was given orally for 7, 14, or 21 days followed by a 14-day treatment-free period. Escalating cohorts of 3-6 patients with advanced solid tumors were treated until DLT was seen in $\geq 2$ patients in a cohort. Serial blood samples were collected for pharmacokinetics and skin biopsies were collected for pharmacodynamics.
\end{abstract}

Results-Sixty-one patients received 5, 10,20,30 or $40 \mathrm{mg}$ once daily for 7 days; 25, 35, 45 or $55 \mathrm{mg} /$ day in four divided doses (QID) for 7 days; or $55,60,70$ or $80 \mathrm{mg} /$ day plus methylphenidate or modafinil with daytime doses (QID/M) for 7-21 days. DLTs of reversible grade 3 benzodiazepine-like effects defined the estimated MTD of $60 \mathrm{mg}$ QID/M for 14 days. MLN8054 was absorbed rapidly, exposure was dose-proportional, and terminal half-life was 30-40 hours. Three patients had stable disease for $>6$ cycles.

Conclusions-MLN8054 dosing for up to 14 days of a 28-day cycle was feasible. Reversible somnolence was dose limiting and prevented achievement of plasma concentrations predicted necessary for target modulation. A recommended dose for investigation in phase 2 trials was not established. A second-generation Aurora A kinase inhibitor is in development.

\section{Keywords}

MLN8054; Aurora A kinase; dose-limiting toxicity; pharmacokinetics; pharmacodynamics

Correspondent E. Claire Dees, MD, UNC Lineberger Comprehensive Cancer Center, University of North Carolina, 170 Manning Drive, 3rd floor POB, CB 7305, Chapel Hill, NC 27599, Telephone: (919) 843-7714, FAX: (919) 966-6735,

claire_dees@med.unc.edu.

${ }^{*}$ Affiliation after study: Forest Research Institute, Inc., Jersey City, NJ

${ }^{\dagger}$ Affiliation after study: Boston University School of Medicine, Boston, MA 


\section{INTRODUCTION}

The Aurora kinases are a family of serine/threonine protein kinases. Three isoforms of Aurora kinase exist (Aurora A, B, and C), each with distinct activities. Aurora A and B have critical roles in the normal progression of cells through mitosis, whereas Aurora $\mathrm{C}$ activity is largely restricted to meiosis. Aurora A kinase localizes to centrosomes and proximal mitotic spindles [1], where it regulates centrosome maturation/separation, the G2-M transition, formation of mitotic spindle poles and spindles, and chromosome alignment and separation [2-5]. Increased Aurora A kinase expression results in oncogenic transformation in preclinical models [6-9] and has been correlated with decreased survival in patients with solid tumors $[10,11]$. Aurora A kinase is amplified and overexpressed in many solid tumors and hematological malignancies [12-16]. Consequently, Aurora A kinase is an attractive target for anticancer treatment [17].

MLN8054 (Figure 1; Millennium, the Takeda Oncology Company) is an orally active small molecule that selectively inhibits Aurora A kinase [18]. MLN8054 induces severe mitotic defects, including delayed progression through mitosis, formation of abnormal mitotic spindles and misaligned chromosomes, and chromosome segregation defects $[18,19]$. MLN8054 led to decreased tumor proliferation in models of human cancer grown in cell culture and antitumor activity in human tumor xenografts including colon, prostate, and lung cancer models [18]. The greatest efficacy was seen with once or twice daily dosing for 21 days in mice, suggesting that prolonged target inhibition results in maximal antitumor activity. In preclinical toxicology studies, dose-limiting toxicities (DLTs) were myelosuppression and gastrointestinal toxicity, and MLN8054 demonstrated high-affinity binding to the alpha-1 subunit of the GABA-A receptor (Data on file, Millennium). Preclinical pharmacokinetic/pharmacodynamic analyses suggest antitumor activity is dosedependent and maintenance of plasma concentrations of $\sim 2000 \mathrm{nM}$ for $8-12$ hours per day is required for efficacy in human tumor xenografts grown in mice [20].

Hepatic biotransformation of MLN8054 was studied in vitro using human liver S9 fractions (Data on file, Millennium). Glucuronidation of the carboxylate moiety of MLN8054 to an acyl glucuronide was the predominant mechanism of biotransformation, Hydroxylation of the azepine moiety of MLN8054 was the major phase 1 biotransformation pathway. Glucuronidation was mediated by UGT1 and UGT2 and hydroxylation by CYP1A2, 2C9, 2C19, 2D6, and 3A4.

This phase 1 study was conducted to: (i) determine the dose-limiting toxicity (DLT) and maximum tolerated dose of MLN8054 when given orally for 7, 14, or 21 days, followed by a 14-day recovery period, the latter thought to be necessary based on neutropenia results from preclinical toxicology studies; (ii) describe the pharmacokinetics of MLN8054 from serial blood samples; (iii) evaluate the relationship between MLN8054 exposure and inhibition of Aurora A kinase in skin basal epithelial cells; and (iv) describe any antitumor activity of MLN8054.

\section{MATERIALS AND METHODS}

\section{Design}

This open-label phase 1 study (NCT00249301) was conducted at 3 centers in the United States between 19 October 2005 and 25 January 2008. The study followed the principles of the Declaration of Helsinki. The protocol was reviewed and approved by the institutional review board at each clinical center. Each patient provided informed written consent prior to enrollment. 


\section{Eligibility}

Patients with a solid tumor malignancy refractory to conventional treatment or for which no standard treatment existed were candidates for this study. Patients were required to be $\geq 18$ years of age and to have an Eastern Cooperative Oncology Group (ECOG) performance status of 0 or 1, expected survival greater than 3 months from study enrollment, and adequate hematologic, renal, and hepatic function. Prior cytotoxic chemotherapy was limited to no more than 4 regimens, and prior radiation therapy must have included less than $25 \%$ of the hematopoietically active bone marrow. Patients were ineligible if they had central nervous system metastases, had undergone peripheral blood stem cell or bone marrow transplantation, or had prior gastrointestinal surgery or conditions that would impair absorption.

\section{Dose Escalation}

The dose escalation scheme is shown in Table 1. In animal studies, dogs were the most sensitive species to MLN8054 and the highest non-severely toxic dose in dogs was $20 \mathrm{mg} /$ $\mathrm{m}^{2} /$ day. One quarter of this dose $\left(5 \mathrm{mg} / \mathrm{m}^{2} /\right.$ day $)$ was chosen as the starting dose for this firstin-human study. Patients were enrolled in escalating dose cohorts of 3-6 patients each; if 1 of 3 patients had a DLT, the cohort was expanded to 6 patients. If $0-1$ of 6 patients experienced a DLT, then dose escalation continued. If $\geq 2$ of 3-6 patients experienced a DLT, then the MTD had been surpassed and a lower dose level or alternate schedule was explored. Dose-escalation decisions incorporated real-time assessment of systemic drug exposure as well as toxicity experience and utilized the Factors of 2 pharmacokinetically guided dose-escalation method [21]. Adverse events (AEs) were defined by CTCAE version 3.0 [22]. A DLT was defined as any of the following during cycle 1: grade 4 neutropenia lasting more than 7 days or associated with fever; grade 4 thrombocytopenia; grade 3 or greater nausea or diarrhea that persisted despite the use of optimal anti-emetic or antidiarrheal therapy; any other grade 3 or greater nonhematologic AE except arthralgia/myalgia or brief ( $<1$ week) fatigue; or any drug-related $\mathrm{AE}$ requiring dose interruption or delay of more than one week.

Patients took MLN8054 orally on an empty stomach, with nothing by mouth 2 hours before and 1 hour after each dose except prescribed medications and water. The initial dosing regimen was once daily for 7 days (QD-7D), with 14-day breaks (21-day cycles). Due to somnolence with the QD regimen, the protocol was amended to include divided four-timesdaily (QID-7D) dosing, with the highest dose at bedtime. The QID administration was designed to minimize daytime sedation (which was not observed in mice) and maximize exposure (supported by PK computer modeling) to potentially therapeutic concentrations of the compound [23]. In later QID cohorts, an oral psychostimulant (methylphenidate or modafinil) was added to daytime doses (QID/M-7D) to further mitigate somnolence. Additional cohorts received extended-duration QID/M dosing for 14-21 days per cycle (QID/M-14D and QID/M-21D), with 14-day breaks (28- and 35-day cycles, respectively).

Adverse event information was collected throughout the study. Safety assessments were based on evaluation of AEs and serious AEs (SAEs), including their potential relationship to the study medication; physical examination; monitoring of clinically significant laboratory tests, including hematologic parameters, liver function tests, and renal function tests; and evaluation of serial electrocardiograms.

\section{Pharmacokinetics}

Blood samples for pharmacokinetic analyses were drawn once at baseline (day 1 predose), serially on day 1 (QD dosing only- $0.5,1,1.5,2,3,4,6,8,10$, and 24 hours postdose) and day 7 (QD dosing—predose and $0.5,1,1.5,2,3,4,6,8,10$, and 24 hours postdose; QID 
dosing — before the second daily dose and $0.5,1,1.5,2,3,4$, and 6 hours postdose); and once daily on days $8-12$.

\section{Pharmacodynamics}

For patients in the QD cohorts, serial 3-mm skin punch biopsies for pharmacodynamic analyses were obtained in a majority of patients $(n=52)$ at baseline and again 6 and 24 hours after the first dose of cycle 1. For patients in the QID cohorts, skin punch biopsies were obtained at baseline and on day 7 before and 2 hours after the second daily dose of MLN8054. The purpose of these biopsies was to detect inhibition of Aurora A kinase in proliferating basal epithelial cells as measured by accumulation of mitotic cells. Immunofluorescence analysis was performed on skin sections, using two mitotic markers, pHisH3 (Cell Signalling Technologies, Danvers, MA) and MPM2 (Cell Signalling Technologies, Danvers, MA). Skin sections were mounted with DAPI Vectashield Hard Set Mounting Medium (Vector Laboratories, Burlingame, CA). The mitotic index was determined by counting the number of mitotic cells per millimeter of the basal epithelial layer.

Before receiving the first dose of MLN8054, patients underwent disease evaluation including physical examination, computed tomography and/or magnetic resonance imaging, and tumor markers when applicable. Evaluations were repeated after every 2 cycles of MLN8054, and disease status was categorized using standard Response Evaluation Criteria in Solid Tumors (RECIST) guidelines [24]. Patients who had stable disease or a partial response continued treatment until there was evidence of disease progression or unacceptable treatment-related toxicity. Patients who tolerated the first cycle of treatment with MLN8054 were allowed to increase the dose of MLN8054 treatment in subsequent cycles of treatment if the higher dose had been found to be tolerable in a subsequent cohort.

\section{Statistical Analysis}

The safety population included all patients who received at least one dose of study drug, while the DLT population included all patients who received study drug at the assigned dose level and had sufficient follow-up to determine if a DLT occurred. Descriptive statistics are reported for baseline values, safety, pharmacokinetics, and pharmacodynamics.

\section{RESULTS}

\section{Patient Characteristics}

A total of 61 patients were treated in 16 dose cohorts (see Table 1) and were evaluable for toxicity. Demographics and baseline characteristics for the safety population are shown in Table 2. The majority of patients were male $(61 \%)$ and median age was 60 years (range, 2480 years). All patients had a Performance Status of $0(54 \%)$ or $1(46 \%)$. The most common primary diagnoses were colorectal cancer $(36 \%)$, lung cancer $(15 \%)$, genitourinary tumors $(13 \%)$, and sarcoma (13\%). Most patients had been heavily pretreated, with $79 \%$ having received 3 or more courses of prior treatment before study entry.

Reasons for discontinuation were progressive disease in 39 patients (64\%), symptomatic deterioration in $9(15 \%)$, AE in $5(8 \%)$, withdrawal of consent in $3(5 \%)$, death of $1(2 \%)$ due to cardiac arrest unrelated to MLN8054, and other reasons in $4(7 \%)$. The median number of MLN8054 treatment cycles was 2 (range, 1-14). Patients received $98 \%$ of all expected doses overall. 


\section{Dose-Limiting Toxicity}

Although 60 unique patients were enrolled in the study, 1 patient was enrolled twice and was separately evaluated for safety as a member of both the QD-7D $40 \mathrm{mg}$ cohort and the QID-7D $25 \mathrm{mg}$ cohort, and thus 61 patients were evaluable for safety. A total of 59 patients (97\%) were evaluable for DLT; the other 2 patients discontinued treatment during cycle 1, before DLT could be evaluated. One patient in the QD-7D $5 \mathrm{mg}$ cohort discontinued due to hospitalization for renal failure and hypovolemia unrelated to MLN8054 and one patient in the QID/M-21D $60 \mathrm{mg}$ cohort discontinued due to hospitalization for spine fracture unrelated to MLN8054.

Table 1 summarizes the observed DLTs by cohort. Somnolence, which resolved in all but one patient, was the only DLT for MLN8054 given without methylphenidate or modafinil. The onset of somnolence and its severity generally were correlated with dose and $\mathrm{C}_{\max }$. Because somnolence was thought to be related to $C_{\max }$, the dosing schedule was changed from daily to QID dosing in an effort to lower peak plasma concentrations and allow further dose escalation. However, dose-limiting somnolence was seen at both QD-7D dosing ( 1 of 6 patients at $30 \mathrm{mg}$ and 2 of 4 patients at $40 \mathrm{mg}$ ) and QID-7D dosing ( 1 of 6 patients at $25 \mathrm{mg}$ and 2 of 4 patients at $55 \mathrm{mg}$ ).

The addition of methylphenidate (e.g. $5 \mathrm{mg}$ oral dose) or modafinil repeated as needed with the three daytime MLN8054 doses allowed further dose escalation. Dose-limiting but reversible somnolence was seen in 1 of 6 patients in the QID/M-7D $60 \mathrm{mg}$ cohort and grade 3 cognitive disorder and hallucination were experienced by 1 of 3 patients in the QID/M-7D $80 \mathrm{mg}$ cohort. The QID/M-7D $80 \mathrm{mg}$ cohort was not expanded to further define the MTD using the 7-day schedule because all three patients at this level experienced grade $\geq 2$ CNS effects and the investigator consensus was to evaluate longer treatment schedules if possible. With 14-day dosing, DLTs of reversible somnolence or other central nervous system effects (fatigue, confusional state, and cognitive disorder) were seen in 2 of 2 patients at QID/ M-14D $70 \mathrm{mg}$. Because these events were seen during the first week of a planned 2-week treatment, subsequent patients were enrolled to a QID/M-14D $60 \mathrm{mg}$ cohort. None of 3 patients in this cohort had a DLT. Using a QID schedule with $10 \mathrm{mg}$ administered 3 times during the day and $30 \mathrm{mg}$ at night, the total daily dose of $60 \mathrm{mg}$ was generally tolerable over a 7- to 14-day schedule.

Of the 4 patients who were enrolled to a QID/M-21D $60 \mathrm{mg}$ cohort, 2 had a DLT. Both patients had a DLT of somnolence and 1 of the patients also had DLTs of cognitive disorder and confusion. Therefore, no additional cohorts were enrolled to 21-day dosing regimens.

Thus, the estimated MTD of MLN8054 was $60 \mathrm{mg}$ divided QID for 7-14 days, given with methylphenidate or modafinil as needed with the daytime doses to manage somnolence.

\section{Adverse Events}

All 61 patients (100\%) were treated and were evaluable for safety. Table 3 summarizes the most frequent drug-related adverse events, which included somnolence, fatigue, confusion, nausea and vomiting. Forty-seven patients $(77 \%)$ experienced drug-related somnolence, with $11(18 \%)$ experiencing grade 3 somnolence. Many of the patients in this study received at least one other medication that could have contributed to somnolence; $37(61 \%)$ received an opioid analgesic, $15(25 \%)$ received a benzodiazepine or other anxiolytic, and $12(20 \%)$ received a hypnotic or sedative agent.

Among the 11 patients with dose-limiting somnolence related to MLN8054 across all dose levels, concomitant use of opioid medication was reported in 8 patients. Opioid use was reported frequently in many patients enrolled to this study, however, and the frequency of 
somnolence was comparable in patients who were or were not receiving concomitant opioids. Moreover, dose-limiting (CTC Grade 3) somnolence was reported in 2 patients without concomitant treatment with opioids or other sedating medications who received the highest MLN8054 dose levels within the first days of dosing, so that further dose escalation was not feasible even in a population not receiving concomitant opioid medications.

Thirty-four patients (56\%) had a grade $\geq 3 \mathrm{AE}$, including 13 patients (21\%) with a drugrelated grade $3 \mathrm{AE}$ (Table 3); no patient had a drug-related grade 4 or $5 \mathrm{AE}$. No dose studied was associated with grade $\geq 3$ mucositis or myelosuppression, predicted to be mechanistic effects associated with Aurora A kinase inhibition.

Nine patients (15\%) died within 21 days of the last dose of MLN8054; none of these deaths were considered drug-related.

\section{Pharmacokinetics}

Forty-eight patients (79\%) had sufficient dosing and MLN8054 concentration-time data to estimate pharmacokinetics. Mean MLN8054 plasma concentration-time profiles for QD-7D doses are shown in Figure 2A. Pharmacokinetic parameters for each cohort are summarized in Table 4. MLN8054 was absorbed over a short period, with $\mathrm{T}_{\max }$ ranging from 1-4 hours. Terminal elimination half-life $\left(\mathrm{t}_{1 / 2}\right)$ was $30-40$ hours. $\mathrm{AUC}_{0-24} \mathrm{hr}$ and $\mathrm{C}_{\max }$ were roughly dose-proportional with QD dosing and the peak-to-trough ratio $\left(\mathrm{C}_{\max } / \mathrm{C}_{\min }\right)$ for all dose levels was approximately 5. Only 1 patient in the QD-7D $30 \mathrm{mg}$ cohort had a trough concentration on day 7 that was $>2000 \mathrm{nM}$, the predicted minimum concentration needed for inhibition of Aurora A kinase based on a tumor xenograft model [20]. The two patients with the highest Day $1 \mathrm{C}_{\max }$ values among PK-evaluable patients enrolled in the QD dosing cohorts both experienced grade 3 somnolence.

The protocol was amended to implement QID-7D dosing to reduce $\mathrm{C}_{\max }$, thereby reducing somnolence, and to increase trough concentrations, thereby increasing the probability of inhibiting Aurora A kinase for a substantial period of time. Using this approach with total daily doses of $25,35,45$, and $55 \mathrm{mg}$, the mean $C_{\max }$ values were $1050,1966,1526$, and $2484 \mathrm{nM}$, respectively, and the mean trough concentrations after the second daily dose on day 7 were $720,1464,1139$, and $1802 \mathrm{nM}$, respectively. The peak-to-trough ratio was between 1.3 and 1.6 across these 4 cohorts, substantially lower than the ratio for QD dosing regimens. On average, drug concentrations for the highest dose, QID-7D $55 \mathrm{mg}$, were close to the target of $2000 \mathrm{nM}$.

Subsequent QID cohorts added oral methylphenidate or modafinil with each of the 3 daytime doses of MLN8054 to mitigate the impact of somnolence. Pharmacokinetic data from the QID/M-7D $70 \mathrm{mg}$ cohort showed promise for maintaining a mean plasma MLN8054 concentration of $2000 \mathrm{nM}$ and a peak-to-trough ratio of 1.65 over the course of treatment.

When the duration of dosing was extended to QID/M-14D, both patients in the $70 \mathrm{mg}$ cohort had a DLT and neither was evaluable for pharmacokinetics. The dose level was reduced to QID/M-14D $60 \mathrm{mg}$ and all 3 patients were evaluable for pharmacokinetics. Mean MLN8054 concentrations in these patients were close to $2000 \mathrm{nM}$ between the second and third doses on day 7, and were $>2000 \mathrm{nM}$ between the second and third doses on day 14 (Figure 2B).

Only 1 patient in the QID/M-21D $60 \mathrm{mg}$ cohort was evaluable for pharmacokinetics on days 14 and 21, and MLN8054 concentrations in this patient were $<2000 \mathrm{nM}$ between the second and third doses on each of these days. Two of the 3 patients with the highest Day $7 \mathrm{C}_{\max }$ values among PK-evaluable patients enrolled in the various QID dosing cohorts experienced 
grade 3 somnolence. One of these patients experienced Grade 3 somnolence despite receiving methylphenidate. Thus, of the 48 patients with sufficient data to estimate pharmacokinetic parameters, 7 patients had trough MLN8054 concentrations that were $>2000 \mathrm{nM}$, including 1 patient in the QD-7D $30 \mathrm{mg}$ cohort, 1 patient in the QID-7D $55 \mathrm{mg}$ cohort, 2 patients in the QID/M-7D $70 \mathrm{mg}$ cohort, 1 patient in the QID/M-7D $80 \mathrm{mg}$ cohort, and 2 patients in the QID/M-14D $60 \mathrm{mg}$ cohort.

\section{Pharmacodynamics}

Skin biopsies were evaluable pre- and post-treatment in 52 patients. Although some patient skin samples had increased numbers of mitotic cells suggestive of Aurora A kinase inhibition after MLN8054 dosing, these increases generally were slight and did not compellingly indicate Aurora A inhibition in any dose cohort. No relationship was observed between the pharmacodynamic parameters and either the MLN8054 dose or the MLN8054 pharmacokinetic parameters. Despite the fact that 7 patients had trough MLN8054 concentrations $>2000 \mathrm{nM}$, the skin biopsies in these patients did not provide significant evidence of Aurora A kinase inhibition.

\section{Clinical Responses}

No complete or partial responses were seen. Nine patients $(15 \%)$ had stable disease for at least 4 cycles, including $3(5 \%)$ with stable disease for greater than 6 cycles. These included 1 patient in the QD-7D $5 \mathrm{mg}$ cohort with colorectal cancer (8 cycles; 165 days), 1 patient in the QID/M-7D $55 \mathrm{mg}$ cohort with extraskeletal chondrosarcoma (8 cycles; 129 days), and another patient in the QID/M-7D $55 \mathrm{mg}$ cohort with spindle-cell sarcoma (12 cycles; 266 days).

\section{DISCUSSION}

As critical regulators of the mitotic process that are frequently overexpressed in human tumors [25], the Aurora kinases have emerged as novel oncologic therapeutic targets. In addition to kinesin spindle (Eg5 motor protein) inhibitors, polo-like kinase inhibitors, and centromeric protein E (CENPE) inhibitors, Aurora kinase inhibitors represent a unique class of antimitotic agents without direct anti-tubulin properties [26]. Compared to taxanes and vinca alkaloids, Aurora kinase inhibitors may improve the therapeutic index by avoiding neurotoxicity and specifically targeting kinases that are only expressed in dividing cells.

Several Aurora kinase inhibitors currently are in phase 1 or 2 testing [27]. Most of these are pan-Aurora inhibitors that inhibit isoforms A, B, and C. MLN8054 is the first selective Aurora A kinase inhibitor to enter clinical trials. Selective inhibition of Aurora A has potential advantages because it is amplified in many solid tumors and hematological malignancies [12-16]. Furthermore, selective Aurora A kinase inhibition may have a different toxicity profile and therapeutic index than pan Aurora inhibitors based upon adverse events specific to inhibiting both Aurora A and Aurora B kinase simultaneously.

This is the first reported experience with an Aurora A kinase inhibitor in clinical testing. This novel oral compound was tolerated well at doses up to $60 \mathrm{mg} /$ day in divided doses. Escalation was halted due to dose-limiting, reversible, benzodiazepine-like somnolence and neurocognitive changes, despite the addition of methylphenidate or modafinil in the higher dose cohorts. MLN8054 is structurally related to the benzodiazepines and as such it has activity against the GABAA $\alpha 1$ receptor. Sedation had been expected from preclinical toxicological evaluation of MLN8054, although it was not anticipated that benzodiazepinelike central nervous system effects would be dose limiting. Because these neurocognitive side effects were thought to be partially dependent on peak plasma concentrations, once- 
daily dosing in the early cohorts was changed to divided daily doses in later cohorts in order to continue dose escalation. Although some patients with somnolence received concomitant treatment with opioids or other sedating medications, somnolence occurred with similar frequency in patients not receiving these medications. In addition, 3 of the patients who experienced dose-limiting somnolence (CTC Grade 3) in Cycle 1 did not receive concomitant treatment with opioids or other sedating medications. These findings indicate that the symptomatic somnolence seen in this study was due to MLN8054.

This phase 1 study also included pharmacokinetic analyses after the first dose in the oncedaily cohorts and at steady state (the second dose on Day 7) in the divided dosing cohorts. MLN8054 was absorbed rapidly, with peak concentrations at 1-4 hours postdose and $t_{1 / 2}$ of 30-40 hours. Drug exposures were roughly dose-proportional in the QD dose range evaluated in this study. The peak-to-trough ratio was reduced from approximately 5 with once-daily dosing to 1.3-1.6 with QID dosing.

A steady state concentration of $2000 \mathrm{nM}$, the concentration estimated to be necessary for antitumor activity, was achieved in some but not all patients at the $60 \mathrm{mg}$ dose, and in few patients in the other dose cohorts. Skin biopsies were evaluated for mitotic arrest in the basal epithelial cells at 24 hours-a surrogate for inhibition of Aurora A. Although some samples had slightly increased numbers of mitotic cells, no clear relationship could be established with either the MLN8054 dose level or MLN8054 concentration across the range of exposures tolerable in this study. The most likely explanation is that prolonged biologically active exposures were not achieved in the patients tested in this study, as subsequent studies using the second-generation Aurora A kinase inhibitor MLN8237 have demonstrated dosedependent pharmacodynamic activity in skin biopsies obtained at similar times using identical assays [28]. Furthermore, there was no consistent evidence of significant myelosuppression or mucositis, the expected anti-mitotic side effects of MLN8054. By contrast, other pan-Aurora kinase inhibitors have reported dose-related neutropenia that defined the MTD $[29,30]$. No patient had a complete or partial response to the doses tested (5-80 mg per day) for 7, 14, or 21 days with 14-day breaks, although stable disease lasting more than 6 cycles was observed in 3 patients, including 1 at the lowest dose tested (QD-7D $5 \mathrm{mg}$ ) and 2 at the highest dose tested without methylphenidate or modafinil support (QID-7D $55 \mathrm{mg}$ ).

In summary, benzodiazepine-like effects, especially somnolence, were DLTs of MLN8054, despite QID dosing and the addition of an oral psychostimulant (methylphenidate or modafinil) with daytime doses. Benzodiazepine-like toxicities were expected, but it was not anticipated that their severity would prevent escalation to doses that provided sustained target plasma concentrations. At the doses studied, there was no evidence of antiproliferative effects such as myelosuppression, mucositis, or tumor response. Despite these limitations, MLN8054 did exhibit several favorable pharmaceutical features, including reliable absorption, dose-proportional systemic exposure, and sufficiently prolonged half life to support a once daily administration schedules. As an anticancer agent, the MLN8054 chemotype is an oral agent distinct from the other Aurora kinase inhibitors currently in development. Building on these results, MLN8237, a second-generation oral Aurora A kinase inhibitor has been developed with structural modifications that are designed to improve the risk-to-benefit profile, particularly with regard to central nervous system effects. Clinical trials of MLN8237 are underway to evaluate the safety, pharmacokinetics, pharmacodynamics, and clinical response in patients with advanced tumors [31,32]. 


\section{Acknowledgments}

The authors thank the following colleagues for their contributions to the study (listed alphabetically): Vijaya Dandamudi, Donna Harper, Gary Hudes, Kimberly Keller, Corey J. Langer, Neal J. Meropol, Patti Murphy, and Sara Ramsey. The authors also thank Michael Cooper for his guidance in the development of MLN8054 and this clinical trial. The authors acknowledge the editorial assistance of Jonathan Latham of PharmaScribe, LLC during the development of this publication, which was funded by Millennium Pharmaceuticals, Inc. This study was supported by Millennium Pharmaceuticals, Inc. Additional support was provided to Fox Chase Cancer Center by grant 5P30CA006927 from the National Cancer Institute and to the University of North Carolina by Grant RR00046 (U54RR024383) from the National Institutes of Health.

\section{References}

1. Crosio C, Fimia GM, Loury R, Kimura M, Okano Y, Zhou H, Sen S, Allis CD, Sassone-Corsi P. Mitotic phosphorylation of histone H3: spatio-temporal regulation by mammalian Aurora kinases. Mol Cell Biol. 2002; 22:874-85. [PubMed: 11784863]

2. Bischoff JR, Plowman GD. The Aurora/Ipl1p kinase family: regulators of chromosome segregation and cytokinesis. Trends Cell Biol. 1999; 9:454-9. [PubMed: 10511710]

3. Carmena M, Earnshaw WC. The cellular geography of aurora kinases. Nat Rev Mol Cell Biol. 2003; 4:842-54. [PubMed: 14625535]

4. Giet R, Prigent C. Aurora/Ipl1p-related kinases, a new oncogenic family of mitotic serine-threonine kinases. J Cell Sci. 1999; 112(Pt 21):3591-601. [PubMed: 10523496]

5. Nigg EA. Mitotic kinases as regulators of cell division and its checkpoints. Nat Rev Mol Cell Biol. 2001; 2:21-32. [PubMed: 11413462]

6. Zhou H, Kuang J, Zhong L, Kuo WL, Gray JW, Sahin A, Brinkley BR, Sen S. Tumour amplified kinase STK15/BTAK induces centrosome amplification, aneuploidy and transformation. Nat Genet. 1998; 20:189-93. [PubMed: 9771714]

7. Goepfert TM, Adigun YE, Zhong L, Gay J, Medina D, Brinkley WR. Centrosome amplification and overexpression of aurora A are early events in rat mammary carcinogenesis. Cancer Res. 2002; 62:4115-22. [PubMed: 12124350]

8. Wang X, Zhou YX, Qiao W, Tominaga Y, Ouchi M, Ouchi T, Deng CX. Overexpression of aurora kinase $\mathrm{A}$ in mouse mammary epithelium induces genetic instability preceding mammary tumor formation. Oncogene. 2006; 25:7148-58. [PubMed: 16715125]

9. Zhang D, Hirota T, Marumoto T, Shimizu M, Kunitoku N, Sasayama T, Arima Y, Feng L, Suzuki M, Takeya M, Saya H. Cre-loxP-controlled periodic Aurora-A overexpression induces mitotic abnormalities and hyperplasia in mammary glands of mouse models. Oncogene. 2004; 23:8720-30. [PubMed: 15480417]

10. Nadler Y, Camp RL, Schwartz C, Rimm DL, Kluger HM, Kluger Y. Expression of Aurora A (but not Aurora B) is predictive of survival in breast cancer. Clin Cancer Res. 2008; 14:4455-62. [PubMed: 18628459]

11. Landen CN Jr, Lin YG, Immaneni A, Deavers MT, Merritt WM, Spannuth WA, Bodurka DC, Gershenson DM, Brinkley WR, Sood AK. Overexpression of the centrosomal protein Aurora-A kinase is associated with poor prognosis in epithelial ovarian cancer patients. Clin Cancer Res. 2007; 13:4098-104. [PubMed: 17634535]

12. Sakakura C, Hagiwara A, Yasuoka R, Fujita Y, Nakanishi M, Masuda K, Shimomura K, Nakamura Y, Inazawa J, Abe T, Yamagishi H. Tumour-amplified kinase BTAK is amplified and overexpressed in gastric cancers with possible involvement in aneuploid formation. Br J Cancer. 2001; 84:824-31. [PubMed: 11259099]

13. Bischoff JR, Anderson L, Zhu Y, Mossie K, Ng L, Souza B, Schryver B, Flanagan P, Clairvoyant F, Ginther C, Chan CS, Novotny M, Slamon DJ, Plowman GD. A homologue of Drosophila aurora kinase is oncogenic and amplified in human colorectal cancers. EMBO J. 1998; 17:305265. [PubMed: 9606188]

14. Yamamoto Y, Matsuyama H, Kawauchi S, Furuya T, Liu XP, Ikemoto K, Oga A, Naito K, Sasaki K. Biological characteristics in bladder cancer depend on the type of genetic instability. Clin Cancer Res. 2006; 12:2752-8. [PubMed: 16675567] 
15. Ginestier C, Cervera N, Finetti P, Esteyries S, Esterni B, Adelaide J, Xerri L, Viens P, Jacquemier J, Charafe-Jauffret E, Chaffanet M, Birnbaum D, Bertucci F. Prognosis and gene expression profiling of 20q13-amplified breast cancers. Clin Cancer Res. 2006; 12:4533-44. [PubMed: 16899599]

16. Sen S, Zhou H, Zhang RD, Yoon DS, Vakar-Lopez F, Ito S, Jiang F, Johnston D, Grossman HB, Ruifrok AC, Katz RL, Brinkley W, Czerniak B. Amplification/overexpression of a mitotic kinase gene in human bladder cancer. J Natl Cancer Inst. 2002; 94:1320-9. [PubMed: 12208897]

17. Gautschi O, Heighway J, Mack PC, Purnell PR, Lara PN Jr, Gandara DR. Aurora kinases as anticancer drug targets. Clin Cancer Res. 2008; 14:1639-48. [PubMed: 18347165]

18. Manfredi MG, Ecsedy JA, Meetze KA, Balani SK, Burenkova O, Chen W, Galvin KM, Hoar KM, Huck JJ, LeRoy PJ, Ray ET, Sells TB, Stringer B, Stroud SG, Vos TJ, Weatherhead GS, Wysong DR, Zhang M, Bolen JB, Claiborne CF. Antitumor activity of MLN8054, an orally active smallmolecule inhibitor of Aurora A kinase. Proc Natl Acad Sci U S A. 2007; 104:4106-11. [PubMed: 17360485]

19. Hoar K, Chakravarty A, Rabino C, Wysong D, Bowman D, Roy N, Ecsedy JA. MLN8054, a small-molecule inhibitor of Aurora A, causes spindle pole and chromosome congression defects leading to aneuploidy. Mol Cell Biol. 2007; 27:4513-25. [PubMed: 17438137]

20. Huck, J.; Chakravarty, A.; Yu, L.; Zhang, M.; Burke, K.; Kim, M-S.; Stringer, B.; Sells, T.; Claiborne, C.; Manfredi, M. Preclinical PK/PD/efficacy relationship of MLN8054, a small molecule Aurora A kinase inhibitor 2007 (Abstract C191). AACR-NCI-EORTC International Conference: Molecular Targets and Cancer Therapeutics; San Francisco, CA. 2007.

21. Collins JM, Zaharko DS, Dedrick RL, Chabner BA. Potential roles for preclinical pharmacology in phase I clinical trials. Cancer Treat Rep. 1986; 70:73-80. [PubMed: 3753662]

22. DCTD, NCI, NIH, DHHS; December 12. 2003 Cancer Therapy Evaluation Program Common Terminology Criteria for Adverse Events, Version 3.0. Available online: http://ctep.cancer.gov/reporting/ctc.html

23. Lee Y, Eton O, Pappas J, Chen S, Paton M, Dees EC, Jones S, Cohen RB, Cervantes A, Tabernero J. Dosing strategies for MLN8054, a selective Aurora A kinase inhibitor, based on pharmacokinetic modeling and simulations. Eur J Cancer Suppl. 2008; 6(12):130-1.

24. Therasse, P.; Arbuck, SG.; Eisenhauer, EA.; Wanders, J.; Kaplan, RS.; Rubinstein, L.; Verweij, J.; Van Glabbeke, M.; van Oosterom, AT.; Christian, MC.; Gwyther, SG. J Natl Cancer Inst. Vol. 92. 2000. New guidelines to evaluate the response to treatment in solid tumors. European Organization for Research and Treatment of Cancer, National Cancer Institute of the United States, National Cancer Institute of Canada; p. 205-16.

25. Keen N, Taylor S. Aurora-kinase inhibitors as anticancer agents. Nat Rev Cancer. 2004; 4:927-36. [PubMed: 15573114]

26. Jackson JR, Patrick DR, Dar MM, Huang PS. Targeted anti-mitotic therapies: can we improve on tubulin agents? Nat Rev Cancer. 2007; 7:107-17. [PubMed: 17251917]

27. Cheung CH, Coumar MS, Hsieh HP, Chang JY. Aurora kinase inhibitors in preclinical and clinical testing. Expert Opin Investig Drugs. 2009; 18:379-98.

28. Cervantes-Ruiperez A, Elez ME, Roselló S, Macarulla T, Rodríguez-Braun E, Lee Y, Ecsedy J, Liu H, Fingert H, Tabernero J. Phase I pharmacokinetic (PK) and pharmacodynamic (PD) study of MLN8237, a novel selective aurora A kinase (AAK) inhibitor, in patients (pts) with advanced solid tumors. J Clin Oncol. 2009; 27(15s) Abstr 2565.

29. Cohen RB, Jones SF, von Mehren M, Cheng J, Spiegel DM, Laffranchi B, Mariani M, Spinelli R, Magazzu D, Burris HA III. Phase I study of the pan aurora kinases (AKs) inhibitor PHA-739358 administered as a $24 \mathrm{~h}$ infusion without/with G-CSF in a 14-day cycle in patients with advanced solid tumors. J Clin Oncol. 2008; 26(15S):a2520.

30. Jones SF, Burris HA III, Dumez H, Infante JR, Fowst C, Gerletti P, Xu H, Jakubczak J, Mellaerts N, Schöffski P. Phase I accelerated dose-escalation, pharmacokinetic (PK) and pharmacodynamic study of PF-03814735, an oral aurora kinase inhibitor, in patients with advanced solid tumors: Preliminary results. J Clin Oncol. 2008; 26(15S):a2517. 
31. Infante J, Dees EC, Cohen RB, Burris H, O’Neil B, Murphy P, Lee Y, Pappas J, Ecsedy JA, Eton O. Phase I study of the safety, pharmacokinetics (PK), and pharmacodynamics (PD) of MLN8237, a selective Aurora A kinase inhibitor, in the United States. Eur J Cancer Suppl. 2008; 6(12):90-91.

32. Tabernero J, Cervantes A, Elez E, Macarulla T, Roselló S, Rodríguez-Braun E, Stringer B, Shinde V, Danaee H, Eton O. MLN8237, an oral selective Aurora A kinase inhibitor: initial results of dose-finding pharmacokinetic-pharmacodynamic phase I study. Eur J Cancer Suppl. 2008; 6(12): 92. 


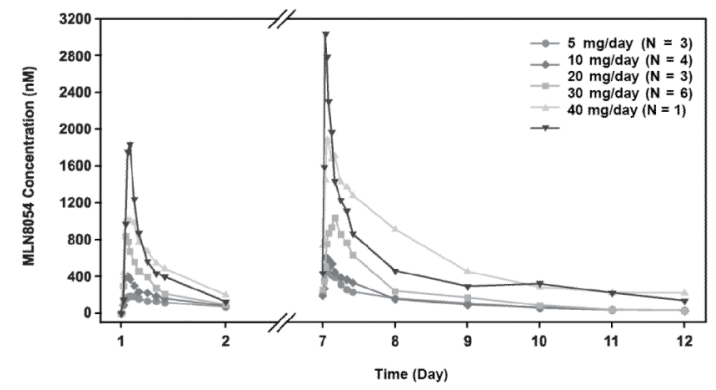

Figure 1.

Chemical Structure of MLN8054 (Reprinted from Manfredi et al (18)). Copyright 2007 National Academy of Sciences, U.S.A. 



Figure 2.

MLN8054 plasma concentration-time profiles. (A) Mean values in the QD-7D cohorts; (B) Individual patients in the QID/M-14D $60 \mathrm{mg}$ cohort. Open symbols = mean values; closed symbols $=$ individual data. 


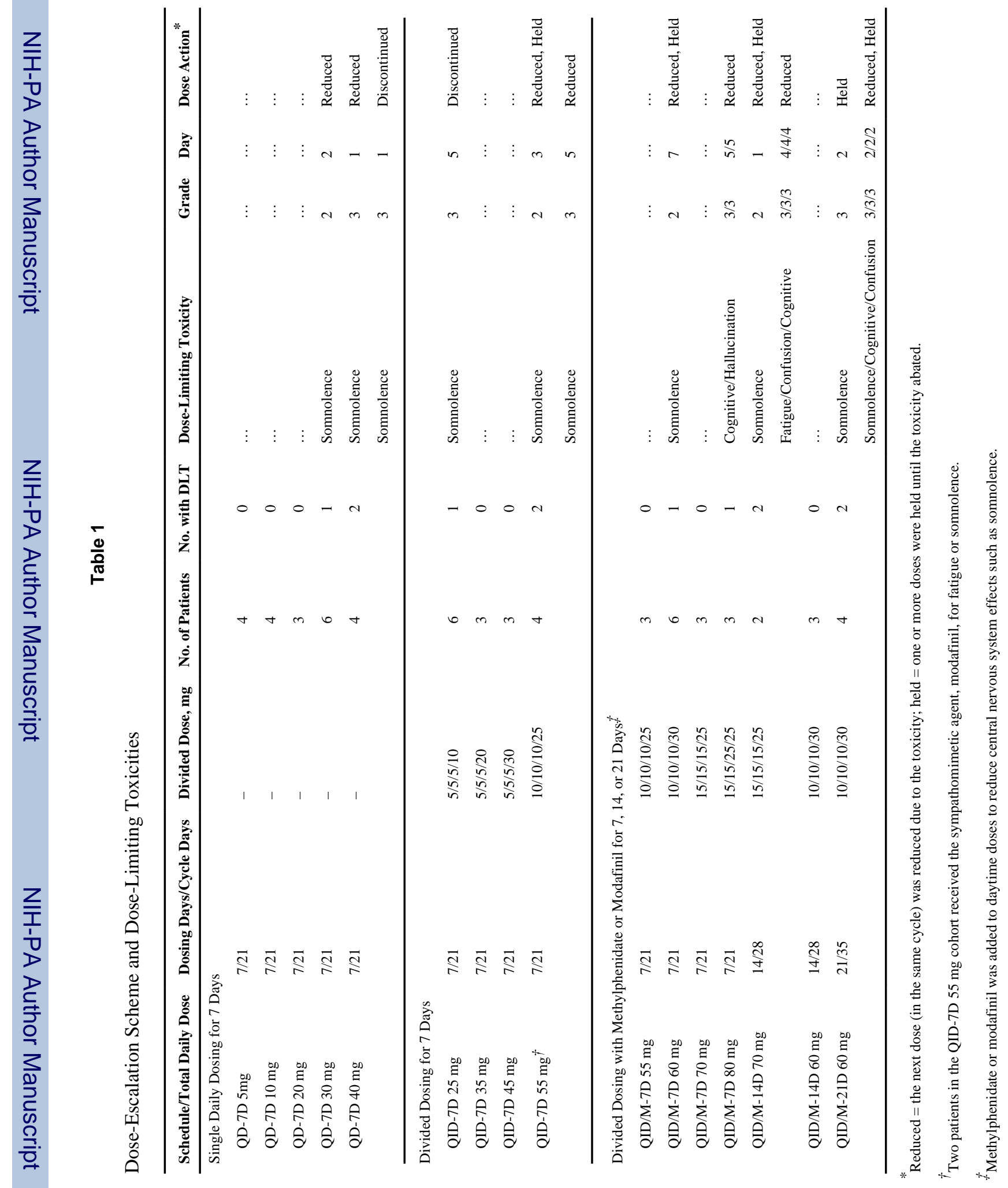



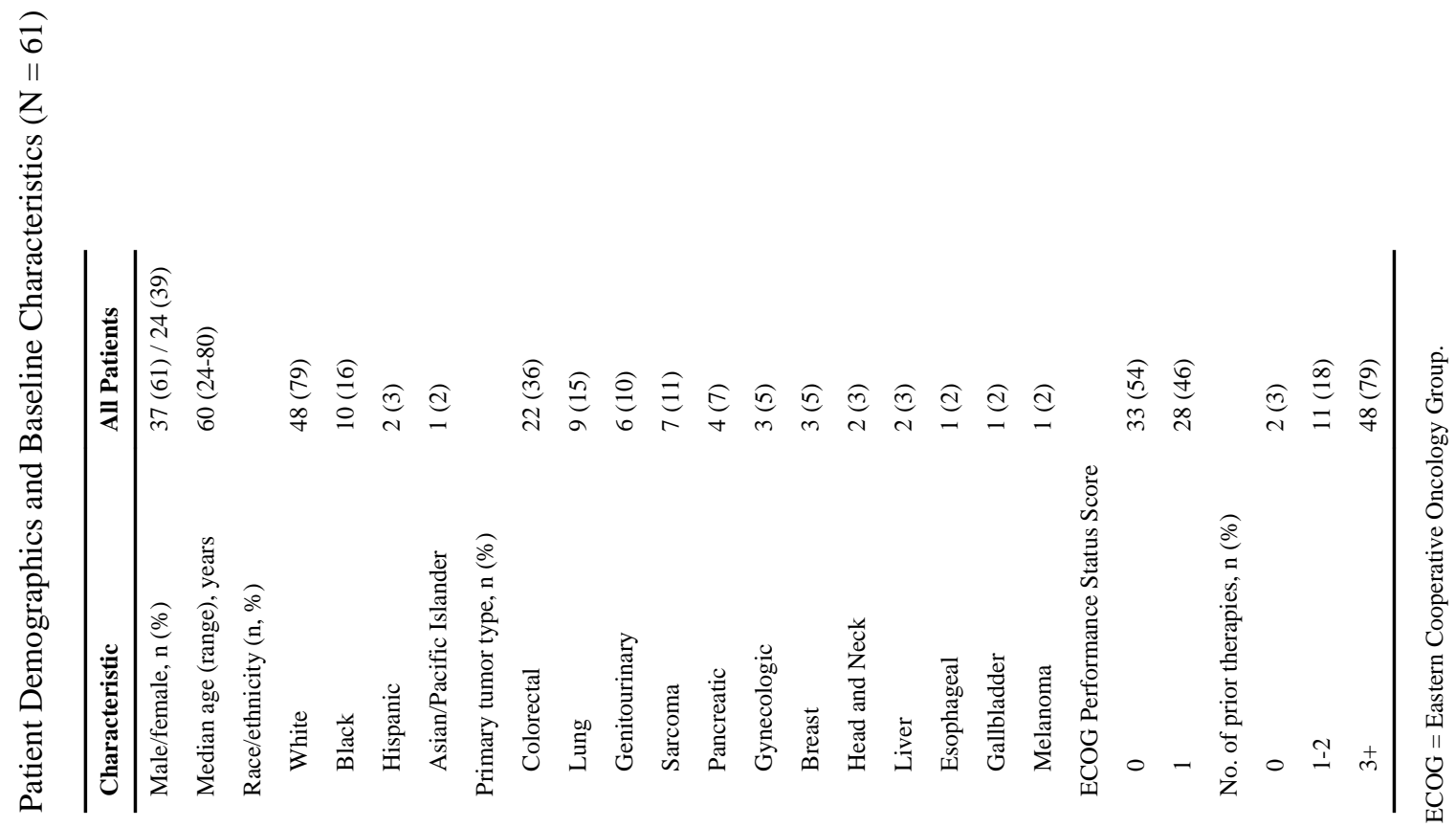


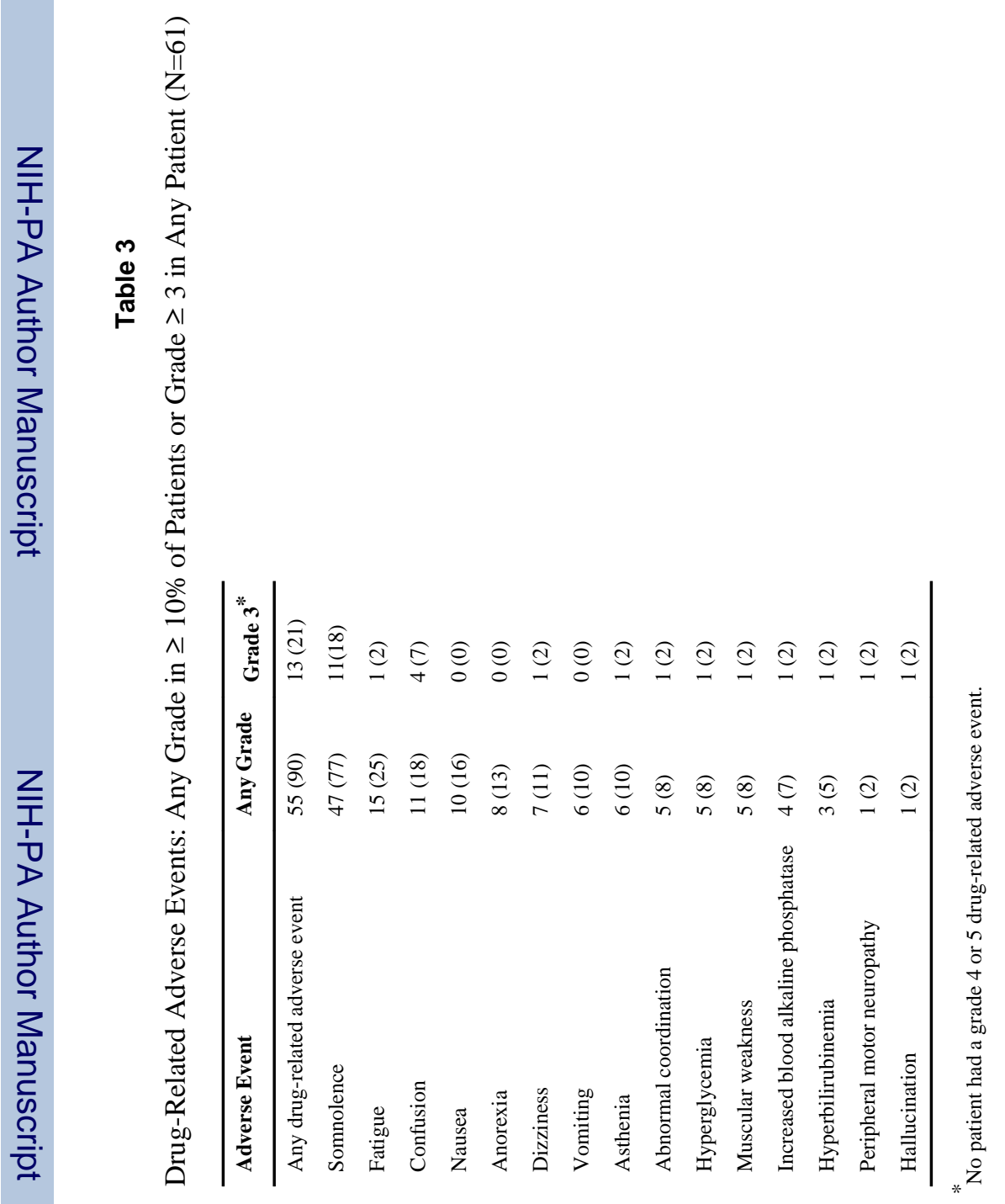

Cancer Chemother Pharmacol. Author manuscript; available in PMC 2012 April 1. 


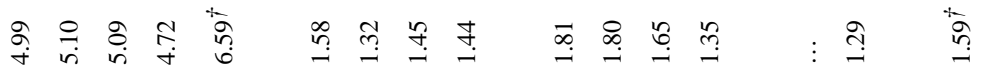

